\title{
FINITENESS AND VANISHING THEOREMS FOR COMPLETE OPEN RIEMANNIAN MANIFOLDS
}

\author{
ZHONGMIN SHEN
}

Let $M^{n}$ denote an $n$-dimensional complete open Riemannian manifold. In [AG] Abresch and Gromoll introduced a new concept of "diameter growth." Roughly speaking, one would like to measure the essential diameter of ends at distance $r$ from a fixed point $p \in M^{n}$. They showed that $M^{n}$ is homotopy equivalent to the interior of a compact manifold with boundary if $M^{n}$ has nonnegative Ricci curvature and diameter growth of order $o\left(r^{1 / n}\right)$, provided the sectional curvature is bounded from below. It is well known that any complete open manifold with nonnegative sectional curvature has finite topological type. This is a weak version of the Soul Theorem of Cheeger-Gromoll [CG]. Examples of Sha and Yang show that this kind of finiteness result does not hold for complete open manifolds with nonnegative Ricci curvature in general (see [SY1, SY2]), and additional assumptions are therefore required.

We will use a concept of the essential diameter of ends slightly stronger than that of [AG]: For any $r>0$, let $B(p, r)$ denote the geodesic ball of radius $r$ around $p$. Let $C(p, r)$ denote the union of all unbounded connected components of $M^{n} \backslash \overline{B(p, r)}$. For $r_{2}>r_{1}>0$, set $C\left(p ; r_{1}, r_{2}\right)=$ $C\left(p, r_{1}\right) \cap B\left(p, r_{2}\right)$. Let $1>\alpha>\beta>0$ be fixed numbers. For any connected component $\Sigma$ of $C\left(p ; \alpha r, \frac{1}{\alpha} r\right)$, and any two points $x, y \in \Sigma \cap \partial B(p, r)$, consider the distance $d_{r}(x, y)=\inf$ Length $(\phi)$ between $x$ and $y$ in $C(p, \beta r)$, where the infimum is taken over all smooth curves $\phi \subset C(p, \beta r)$ from $x$ to $y$. Set $\operatorname{diam}(\Sigma \cap \partial B(p, r), C(p, \beta r))=\sup d_{r}(x, y)$, where $x, y \in \Sigma \cap \partial B(p, r)$. Then the diameter of ends at distance $r$ from $p$ is defined by

$$
\operatorname{diam}(p, r)=\sup \operatorname{diam}(\Sigma \cap \partial B(p, r), C(p, \beta r)),
$$

where the supremum is taken over all connected components $\Sigma$ of $C\left(p ; \alpha r, \frac{1}{\alpha} r\right)$. The diameter defined here is not smaller than that defined by Abresch and Gromoll. Our definition will be essential in Lemma 3 and its applications.

The purpose of this note is to announce the following results.

THEOREM A. Let $M$ be a complete open Riemannian manifold with sectional curvature $K_{M} \geq-K^{2}$ for some constant $K>0$. Assume that for some base point $p \in M$,

$$
\limsup _{r \rightarrow+\infty} \operatorname{diam}(p, r)<\frac{\ln 2}{K} .
$$

Received by the editors February 6, 1989 and, in revised form, May 25, 1989. 1980 Mathematics Subject Classification (1985 Revision). Primary 53C20. 
Then $M$ is homotopy equivalent to the interior of a compact manifold with boundary.

THEOREM B. Let $M^{n}$ be an n-dimensional complete open Riemannian manifold. Suppose that the sectional curvature $K_{M} \geq-K^{2}$ for some constant $K>0$. Assume that for some $2 \leq k \leq n-1, M^{n}$ has nonnegative $k$ th-Ricci curvature and that for some $p \in M^{n}$,

$$
\limsup _{r \rightarrow+\infty} \frac{\operatorname{diam}(p, r)}{r^{\frac{1}{k+1}}}<\left[\frac{2(k+1)}{k}\left(\frac{(k-1) \ln 2}{2 k K}\right)^{k}\right]^{1 /(k+1)} .
$$

Then $M^{n}$ is homotopy equivalent to the interior of a compact manifold with boundary.

TheOREM C. Let $M^{n}$ be an n-dimensional complete open Riemannian manifold. Assume that for some $1 \leq k \leq n-1, M^{n}$ has positive kth-Ricci curvature everywhere and that for some $p \in M^{n}, M^{n}$ has diameter growth of order $o(r)$, i.e.

$$
\limsup _{r \rightarrow+\infty} \frac{\operatorname{diam}(p, r)}{r}=0 .
$$

Then $M^{n}$ has the homotopy type of a $C W$-complex with cells of dimensions $\leq k-1$.

The precise condition that $M^{n}$ have nonnegative (positive) $k$ th-Ricci curvature at some point $x \in M^{n}$ is that for all $v$ in the span of any orthonormal set $\left\{e_{1}, \ldots, e_{k+1}\right\}$ in $T_{x} M^{n}$,

$$
\sum_{i=1}^{k+1}\left\langle R\left(e_{i}, v\right) v, e_{i}\right\rangle \geq 0(>0),
$$

where $R(x, y) z$ denotes th curvature tensor of $M^{n}$ (cf. also [H] for the definition of $k$ th-Ricci curvature).

REMARK 1. (1) In Theorem A the upper bound $\ln 2 / K$ must depend on $K$. Otherwise, the connected sum of infinitely many copies of $S^{2} \times S^{2}$ (see [AG]) provides an easy counterexample.

(2) Theorem B generalizes the Abresch-Gromoll Theorem [AG].

(3) The condition in Theorem C can be weakened to that $M^{n}$ has nonnegative $k$ th-Ricci curvature everywhere and positive $k$ th-Ricci curvature outside a compact subset of $M^{n}$ (see Lemma 5).

(4) The same argument as in [AG] shows that any complete open Riemannian manifold with nonnegative Ricci curvature must have diameter growth of order $o(r)$. We do not know whether the condition in Theorem $\mathrm{C}$ on diameter growth is necessary. Examples in [SY1, SY2, We and GM] have diameter growth of order at most $o(r)$.

It is a pleasure to thank D. Gromoll for some valuable suggestions. I would also like to thank G. Gong, A. Phillips and G. Wei for helpful discussions.

OutLine OF Proofs. Throughout this part we assume that $M^{n}$ denotes a complete open Riemannian manifold of dimension $n$ and $p$ is a point of 
$M^{n}$ fixed during the discussion. For arbitrary $t \geq 0$, let $R_{t}(p)=\{\gamma(t) ; \gamma$ is a ray emanating from $p\}$, which is a closed subset of the distance sphere $S(p, t)$. Set $B_{p}^{t}(x)=t-d\left(x, R_{t}(p)\right)$ for any $x \in M^{n}$. It is easy to see that $B_{p}^{t}(x)$ is increasing in $t$ and $\left|B_{p}^{t}(x)\right| \leq d(p, x)$ for any $x \in M^{n}$. The generalized Busemann function $B_{p}$ is defined as $B_{p}(x)=\lim _{t \rightarrow+\infty} B_{p}^{t}(x)$, which is a Lipschitz function with Lipschitz constant 1 . The excess function $E_{p}$ is defined as $E_{p}(x)=d(p, x)-B_{p}(x)$. We will introduce a new function $L_{p}$ which plays an essential role in the study of the generalized Busemann function $B_{p}$. Set $L_{p}(x)=d\left(x, R_{t}(p)\right)$, where $t=d(p, x)$. Since $B_{p}^{t}(x)$ is increasing in $t$, it is easy to see that $E_{p}(x) \leq L_{p}(x)$ and $d(p, x)-L_{p}(x) \leq B_{p}(x)$ for all $x \in M^{n}$. A more detailed discussion for generalized Busemann functions has been given by $\mathrm{H}$. Wu [W1]. For the purpose of this note, we need the following

LEMMA 1. For any $q \in M^{n}$, there exists a ray $\sigma_{q}(t)$ emanating from $q$ such that for all $t \geq 0$, the function $B_{p}^{q, t}(x)$ defined by $B_{p}(q)+t-d\left(x, \sigma_{q}(t)\right)$ supports $B_{p}(x)$ at $q$, namely $B_{p}^{q, t}(x) \leq B_{p}(x)$ for all $x \in M^{n}$ and $B_{p}^{q, t}(q)=$ $B_{p}(q)$.

LEMMA 2. Suppose that $M^{n}$ has sectional curvature $K_{M} \geq-K^{2}$ for some $K>0$, then for any critical point $q$ with respect to $p$,

$$
E_{p}(q) \geq \frac{1}{K}\left(\frac{e^{K d(p, q)}}{\cosh K d(p, q)}\right) .
$$

Notice that $E_{p}(x) \leq L_{p}(x)$ for all $x \in M^{n}$. Thus if $\lim \sup _{d(p, x) \rightarrow+\infty}$ $L_{p}(x)<\frac{\ln 2}{K}$, Lemma 2 shows that outside a compact subset there is no critical point with respect to $p$, Theorem A follows from this argument and the following

Lemma 3. Suppose that $M^{n}$ has diameter growth of order $o(r)$. Then there exists an $R>0$ such that for any $x \in M^{n} \backslash B(p, R)$,

$$
L_{p}(x) \leq \operatorname{diam}(p, d(p, x)),
$$

and the Busemann function $B_{p}$ is proper.

Notice that $d(p, x)-L_{p}(x) \leq B_{p}(x)$ for all $x \in M^{n}$. It is clear that (1) implies that $g(x) \equiv d(p, x)-L_{p}(x)$ is proper, and so is $B_{p}(x)$.

One can obtain a better estimate for $E_{p} \leq L_{p}$ in terms of $L_{p}$ if $M^{n}$ has nonnegative $k$ th-Ricci curvature.

Lemma 4. Suppose that $M^{n}$ has nonnegative kth-Ricci curvature for some $2 \leq k \leq n-1$, then for all $x \in M^{n}$ with $L_{p}(x)<d(p, x)$,

$$
E_{p}(x) \leq \frac{2 k}{k-1}\left[\frac{k}{2(k+1)} \times \frac{L_{p}(x)^{k+1}}{d(p, x)-L_{p}(x)}\right]^{1 / k} .
$$

The proof of Lemma 4 depends on Lemma 1 and the maximum principle. Theorem B therefore follows from Lemmas 2, 3, and 4. For the proof of Theorem C, we need Lemma 3 and the following 
LEMMA 5. Suppose that for some $1 \leq k \leq n-1, M^{n}$ has nonnegative $k$ th-Ricci curvature everywhere and positive kth-Ricci curvature outside a compact subset. If the Busemann function $B_{p}$ is proper, then there exists a $C^{2}$ function $\chi(t)$ such that $\chi \circ B_{p}$ is proper and strictly $k$-convex. Therefore $M^{n}$ has the homotopy type of a $C W$-complex with cells of dimensions $\leq$ $k-1$.

Compare [W2] for a definition of $k$-convexity. It seems to be crucial that the Busemann function $B_{p}$ is proper. The first assertion in Lemma 5 follows from Lemma 1 . If we assume that $\chi \circ B_{p}$ is proper and strictly $k$ convex, then the last assertion in Lemma 5 follows from Wu's Smoothing Theorem [W2] and the standard Morse Theory [M]. This proves Theorem C.

REMARK 2. An observation of Cheeger-Gromoll ([CG], sharpened in [GW]) is that if $M^{n}$ has nonnegative sectional curvature outside a compact subset, then $M^{n}$ has finite topological type and $B_{p}$ is a proper function. If an addition, $M^{n}$ has nonnegative $k$ th-Ricci curvature everywhere and positive $k$ th-Ricci curvature outside a compact subset, then $M^{n}$ has the homotopy type of a CW-complex with finitely many cells of dimensions $\leq k-1$ (cf. [W2]).

\section{REFERENCES}

[AG] U. Abresch and D. Gromoll, On complete manifolds with nonnegative Ricci curvature, Preprint.

[CG] J. Cheeger and D. Gromoll, On the structure of complete manifolds of nonnegative curvature, Ann. of Math. (2) 96 (1974), 413-443.

[GM] D. Gromoll and W. T. Meyer, Examples of complete manifolds with positive Ricci curvature, J. Differential Geom. 21 (1985), 195-211.

[GW] R. Greene and $\mathrm{H}$. Wu, Integrals of subharmonic functions on manifolds of nonnegative curvature, Invent. Math. 27 (1974), 265-298.

[H] P. Hartman, Oscillation criteria for self-adjoint second-order differential systems and "principal sectional curvature", J. Differential Equations 34 (1979), 326-338.

[M] J. Milnor, Morse theory, Princeton Univ. Press, Princeton, N.J., 1975.

[SY1] J. Sha and D. Yang, Examples of manifolds of positive Ricci curvature, J. Differential Geom. (to appear).

[SY2] _ Positive Ricci curvature on the connected sums of $S^{n} \times S^{m}$, Preprint.

[W1] H. Wu, An elementary method in the study of nonnegative curvature, Acta Math. 142 (1979), 57-78.

[W2] __, Manifolds of partially positive curvature, Indiana Univ. Math. J. 36 (1987), 525-548.

[We] G. Wei, Examples of complete manifolds of positive Ricci curvature with nilpotent isometry groups, Bull. Amer. Math. Soc. (N.S.) 19 (1988), 311-313.

Department of Mathematics, SUNY at Stony Brook, Stony Brook, New York, 11794 